Pacific Journal of Mathematics

SUBMANIFOLDS OF ACYCLIC 3-MANIFOLDS 


\title{
SUBMANIFOLDS OF ACYCLIC 3-MANIFOLDS
}

JoŽE VRABEC

\begin{abstract}
It is proved that, from the viewpoint of "geometric" homology theory, an arbitrary embedding of a closed surface $S$ in any 3-manifold with trivial first homology group looks exactly like the standard embedding of $S$ in the euclidean 3-space. A consequence: every compact subset of a 3-manifold with trivial first homology group can be embedded in a homology 3-sphere. Necessary and sufficient (homological) conditions are given for a compact 3-manifold to be embeddable in some acyclic 3-manifold (or in some homology 3-sphere).
\end{abstract}

\section{Definitions and preliminaries.}

Manifolds. We work in the $P L$ category. Each manifold is supposed to have a fixed $P L$ structure. If $M$ is a manifold, then by a submanifold of $M$ or by a surface, simple closed curve, arc, etc., in $M$ we always mean a respective object contained in $M$ as a subpolyhedron (in the chosen $P L$ structure of $M$ ). All maps are assumed to be $P L$. Our manifolds are never automatically assumed to be without boundary, compact, connected, or orientable. However, by a surface we mean a compact, connected, orientable 2-manifold. A cube with $n$ handles is a 3-manifold homeomorphic to a regular neighborhood of a connected finite linear graph of Euler characteristic $1-n$ in $E^{3}$.

We denote the interior of a manifold $M$ by int $M$ and the boundary by $\mathrm{Bd} M$. However, if $M$ is oriented, then by $\partial M$ we denote the manifold $\mathrm{Bd} M$ oriented coherently with $M$. The symbol $\partial$ also denotes the boundary in the homological sense. Let $M$ be an oriented manifold and $P$ a codimension 0 submanifold of $M$. Whenever we talk of $P$ as an oriented manifold, we assume that $P$ has the orientation inherited from $M$, unless explicitly stated otherwise. If $M$ is an oriented manifold, then $M$ with the opposite orientation is sometimes denoted by $-M$.

Homology. All homology and cohomology groups, cycles, chains, etc., have integer coefficients. If $z_{1}, z_{2}$ are $n$-cycles in a space $X$, then $z_{1} \sim z_{2}$ means " $z_{1}$ is homologous to $z_{2}$ ". A compact oriented $n$-submanifold $N$ of an $m$-manifold $M$ generates a uniquely determined $P L n$-chain in $M$. This chain is a cycle if and only if $N$ is a closed manifold. We shall make no distinction in notation between $N$ and the $n$-chain it represents. If $M$ is a manifold of dimension 
at least 2, then every element of $H_{1}(M)$ can be represented by an oriented closed 1-manifold in int $M$. If $M$ is a 3-manifold, if $J \subset M$ is a closed oriented 1-manifold, and if $J \sim 0$ in $M$, then there exists a compact oriented 2-manifold $F$ in $M$ such that $J=\partial F$.

If $X, Y$ are spaces and $f: X \rightarrow Y$ a map, then by $f_{*}$ we denote the homomorphism $H_{1}(X) \rightarrow H_{1}(Y)$ induced by $f$.

Let $S$ be an oriented 2-manifold and $x, y$ either two 1-cycles in $S$ or two elements of $H_{1}(S)$. By $\operatorname{sc}(x, y)$ we denote the (integral) intersection number of $x$ and $y$. The following is well-known.

Lemma 1.1. Let $M$ be an oriented 3-manifold and let $J, K$ be closed oriented 1-manifolds in $\partial M$. If $J \sim K \sim 0$ in $M$, then $\operatorname{sc}(J, K)=0$.

A polyhedron $X$ is acyclic if it is connected and has $H_{n}(X)=0$ for $n>0$. We will call $X 1$-acyclic if it is connected and has $H_{1}(X)=0$. Note that any 1-acyclic manifold $W$ is orientable. The reason is that $\pi_{1}(W)$ contains no subgroups of index 2; a subgroup of index 2 would contain the commutator subgroup of $\pi_{1}(W)$, but the commutator subgroup is the whole $\pi_{1}(W)$ since $H_{1}(W)=0$. A homology $n$-sphere is an $n$-manifold whose homology is isomorphic to the homology of the $n$-sphere. An $n$-manifold will be called subacyclic if it can be embedded in an acyclic $n$-manifold.

2-Manifolds. We give the definition of oriented piping (in dimension 2). Let $S$ be a 2-manifold and $J, K \subset S$ two disjoint oriented simple closed curves. Let $A \subset S$ be an arc from a point $x \in J$ to a point $\mathrm{y} \in K$; let int $\mathrm{A} \subset \operatorname{int} S-(J \cup K)$. Take a regular neighborhood $N$ of $A$ in $S$. The intersection $N \cap J$ is a small arc $J_{0} \subset J$ containing $x$ in its interior. Similarly, $N \cap K$ is an arc $K_{0} \subset K$ with $y \in \operatorname{int} K_{0}$. Let $D$ be the closure of the component of $N-(J \cup K)$ which contains $\operatorname{int} A$. Then $D$ is a disk and $\mathrm{Bd} D$ consists of $J_{0}, K_{0}$, and two "long" $\operatorname{arcs}$ in $\mathrm{Bd} N$. Suppose that $D$ can be oriented coherently with both $J_{0}$ and $K_{0}$. Then the simple closed curve $L=(J \cup K \cup \operatorname{Bd} D)-\operatorname{int}\left(J_{0} \cup K_{0}\right)$ can be oriented so that it induces in $J$-int $J_{0}$ the same orientation as $J$ and in $K-\operatorname{int} K_{0}$ the same orientation as $K$. If this is the case, we say that the oriented simple closed curve $L$ is obtained from $J \cup K$ by piping along $A$ (or that $L$ is obtained by piping $J$ to $K$ or by piping together $J$ and $K$ ). If we think of $J, K, L$ as 1 -cycles and of $D$ as a 2-chain, then $J+K-L=\partial D$. Hence $L \sim$ $J+K$. The following lemma is obvious.

LEMMA 1.2. If $S$ is an oriented surface, then any two components $J$ and $K$ of $\partial S$ can be piped together along any properly embedded arc $A \subset S$ which joins $J$ and $K$. 
For a compact 2-manifold $S$ we define the genus of $S$ to be the sum of genera of the components of $S$.

Groups. If $G$ and $H$ are groups, then $G \approx H$ means " $G$ is isomorphic to $H$ ". Since we deal only with abelian groups we use the term "free group" in the meaning "free abelian group". Let $G$ be a free (abelian) group. We will call $x \in G$ a basic element of $G$ if $x$ is a member of some basis of $G$. Using standard facts we can prove that $x$ is basic if and only if the subgroup of $G$ generated by $x$ is a nonzero direct summand of $G$, or, if and only if $x$ is not equal to $n y$ for any integer $n>1$ and any $y \in G$.

Matrices. If $\boldsymbol{A}$ is any matrix, let $\boldsymbol{A}^{\prime}$ denote the transposed of $\boldsymbol{A}$. For any positive integer $n$ we denote by $\boldsymbol{I}_{n}$ and $\boldsymbol{O}_{n}$ the identity and zero $n \times n$ matrices, respectively. If $n=2 m$, let $J_{n}$ be the matrix

$$
\boldsymbol{J}_{n}=\left[\begin{array}{rr}
\boldsymbol{O}_{m} & \boldsymbol{I}_{m} \\
-\boldsymbol{I}_{m} & \boldsymbol{O}_{m}
\end{array}\right]
$$

For any two integers $i, j$ let $\delta_{i j}$ denote the Kronecker symbol: $\delta_{i j}=1$ if $i=j$ and $\delta_{i j}=0$ otherwise.

2. Surfaces in 1-acyclic 3-manifolds. The main result of this section is the following theorem, which is (together with 2.13 and 2.14) an extension of Theorem 32.3 in [2]. Note that if $S$ is a closed 2-manifold in the interior of a 1-acyclic 3-manifold $W$, then $S$ separates $W$. The reason is that every simple closed curve in $W$ bounds modulo 2 in $W$ and has therefore zero intersection number modulo 2 with $S$. Also, since $W$ is orientable and $S$ separates $W, S$ is necessarily orientable.

THEOREM 2.1. Let $W$ be a 1-acyclic 3-manifold and $S$ a closed surface of genus $g$ in int $W$. Denote by $U$ and $V$ the closures of the two components of $W-S$. Then there exist oriented simple closed curves $J_{1}, \cdots, J_{g}, K_{1}, \cdots, K_{g}$ in $S$ such that

(1) $J_{i}$ and $K_{i}$ intersect transversely at a single point, for each $i$, and $J_{i} \cap J_{j}=J_{i} \cap K_{j}=K_{i} \cap K_{j}=\varnothing$ if $i \neq j$;

(2) $J_{i} \sim 0$ in $U$ and $K_{i} \sim 0$ in $V(i=1, \cdots, g)$;

(3) the homology classes of $J_{1}, \cdots, J_{g}$ form a free basis of $H_{1}(V)$ and the homology classes of $K_{1}, \cdots, K_{g}$ form a free basis of $H_{1}(U)$.

The situation described by this theorem reminds us of the standard embedding of $S$ in $E^{3}$; in fact, the only difference is that 
in the latter case we can choose $J_{1}, \cdots, J_{g}, K_{1}, \cdots, K_{g}$ so that each $J_{i}$ bounds a disk (not only an orientable surface) in $U$ and each $K_{i}$ bounds a disk in $V$.

We postpone the proof of 2.1, which will occupy most of this section, and first prove two consequences of 2.1.

Theorem 2.2. Let $W$ be a 1-acyclic 3-manifold and $S$ a closed surface of genus $g$ in int $W$. Denote by $U$ and $V$ the closures of the two components of $W-S$. Let $V^{\prime}$ be a cube with $g$ handles. Then there exists a homeomorphism $h: \mathrm{Bd} V^{\prime} \rightarrow S$ such that

(1) the 3-manifold $W^{\prime}=V^{\prime} \cup_{h} U$ is 1-acyclic;

(2) if $J$ is a closed oriented 1-manifold in $S$, then $J \sim 0$ in $V$ if and only if $h^{-1}(J) \sim 0$ in $V^{\prime}$.

Proof. Assume 2.1. Think of $V^{\prime}$ as embedded in $E^{3}$; let $S^{\prime}=$ Bd $V^{\prime}$ and $U^{\prime}=E^{3}-$ int $V^{\prime}$. Let $J_{i}, K_{i}(i=1, \cdots, g)$ be oriented simple closed curves in $S$ satisfying the conclusions of 2.1. Let $J_{i}^{\prime}, K_{i}^{\prime} \subset S^{\prime}$ have analogous meaning (with respect to $U^{\prime}$ and $V^{\prime}$ ). Then there exists a homeomorphism $h: S^{\prime} \rightarrow S$ which maps each $J_{i}^{\prime}$ onto $J_{i}$ and each $K_{i}^{\prime}$ onto $K_{i}$ (not necessarily in an orientation preserving way). Let $W^{\prime}=V^{\prime} \cup_{h} U$.

As is well-known, (1) of 2.1 implies that the homology classes of $J_{1}, \cdots, J_{g}, K_{1}, \cdots, K_{g}$ form a basis of $H_{1}(S)$. The homology classes of $K_{1}, \cdots, K_{g}$ belong to the kernel of $H_{1}(S) \rightarrow H_{1}(V)$; it follows from 2.1 (3) that no nontrivial linear combination of the $J_{i}$ is homologous to 0 in $V$. Therefore, a 1-cycle in $S$ bounds in $V$ if and only if it is homologous in $S$ to a linear combination of $K_{1}, \cdots, K_{g}$. Similarly, a 1-cycle in $S^{\prime}$ bounds in $V^{\prime}$ if and only if it is homologous in $S^{\prime}$ to a linear combination of $K_{1}^{\prime}, \cdots, K_{g}^{\prime}$. Therefore, (2) of 2.2 follows directly from the choice of $h$.

To prove (1) of 2.2 choose an arbitrary $x \in H_{1}\left(W^{\prime}\right)$. We have to show that $x=0$. Since $S$ is connected and separates $W^{\prime}, x$ can be represented by a $\operatorname{sum} z_{1}+z_{2}$ where $z_{1}$ is a 1-cycle in $U$ and $z_{2}$ is a 1-cycle in $V^{\prime}$. By $2.1(3), z_{1}$ is homologous in $U$ to a linear combination of $K_{1}, \cdots, K_{g}$ and $z_{2}$ is homologous in $V^{\prime}$ to a linear combination of $J_{1}^{\prime}, \cdots, J_{g}^{\prime}$. Since the sewing map $h$ was chosen so that each $J_{i}^{\prime} \sim 0$ in $U$ and each $K_{i} \sim 0$ in $V^{\prime}, z_{1}+z_{2} \sim 0$ in $W^{\prime}$.

THEOREM 2.3. If $C$ is a compact subset of a 1-acyclic 3-manifold $W$, then $C$ can be embedded in a homology 3-sphere (and thus also in an acyclic 3-manifold unless $C$ is itself a homology 3-sphere).

Proof. We may assume that $C \subset$ int $W$. Cover $C$ by a compact connected 3-submanifold $M$ of int $W$. Take a boundary component $S$ 
of $M$. Denote by $U$ and $V$ the closures of the components of $W-S$; let $U$ be the one which contains $M$. By 2.2 we can replace $V$ by a cube with handles, $V^{\prime}$, in such a way that $W^{\prime}=U \cup V^{\prime}$ is still 1-acyclic. If we perform a similar surgery along each boundary component of $M$, we end up with $M$ embedded in a closed 1-acyclic 3-manifold, $\Sigma$ say. It follows from Poincare duality that $\Sigma$ is a homology 3-sphere. If $C$ is not a closed 3-manifold, then there is a point $p \in \Sigma-C$ and hence $C$ lies in the acyclic 3-manifold $\Sigma-p$.

Before we start proving Theorem 2.1 we establish some homological properties of surfaces. Let $S$ be a closed oriented surface and let $a_{1}, \cdots, a_{n} \in H_{1}(S)$. The intersection number matrix or the sc-matrix of the ordered $n$-tuple $\left(a_{1}, \cdots, a_{n}\right)$ is the $n \times n$ matrix $\boldsymbol{A}=$ $\left(a_{i j}\right)$, where $a_{i j}=\operatorname{sc}\left(a_{i}, a_{j}\right)$. Obviously $\boldsymbol{A}$ is skew-symmetric. The following lemma is proved by a straightforward computation.

Lemma 2.4. Let $S$ be a closed oriented surface and $a_{1}, \cdots, a_{m}$, $b_{1}, \cdots, b_{n} \in H_{1}(S)$. Let $\boldsymbol{A}$ be the sc-matrix of $\left(a_{1}, \cdots, a_{m}\right)$ and $\boldsymbol{B}$ the sc-matrix of $\left(b_{1}, \cdots, b_{n}\right)$. Suppose that there exists an $m \times n$ matrix $\boldsymbol{T}$ with integer entries such that the column vector $\left(a_{1}, \cdots, a_{n}\right)^{\prime}$ is the product of $\boldsymbol{T}$ with the column vector $\left(b_{1}, \cdots, b_{n}\right)^{\prime}$. Then $\boldsymbol{A}=\boldsymbol{T} \boldsymbol{B} \boldsymbol{T}^{\prime}$.

LEMmA 2.5. Let $S$ be a closed oriented surface of genus g. Let $a_{1}, \cdots, a_{2 g} \in H_{1}(S)$ and let $\boldsymbol{A}$ be the sc-matrix of $\left(a_{1}, \cdots, a_{2 g}\right)$. Then $\left\{a_{1}, \cdots, a_{2 g}\right\}$ is a basis of $H_{1}(S)$ if and only if $\operatorname{det} \boldsymbol{A}=1$.

Proof. It is well-known that $H_{1}(S)$ is free of rank $2 g$ and that it has a basis $\left\{b_{1}, \cdots, b_{2 g}\right\}$ whose sc-matrix is $J_{2 g}$. There exists a $2 g \times 2 g$ matrix $\boldsymbol{T}$ with integer entries such that $\left(a_{1}, \cdots, a_{2 g}\right)^{\prime}$ is the product of $\boldsymbol{T}$ with $\left(b_{1}, \cdots, b_{2 g}\right)^{\prime}$. From 2.4 we obtain $\operatorname{det} \boldsymbol{A}=(\operatorname{det} \boldsymbol{T})^{2}$. Obviously $\left\{a_{1}, \cdots, a_{2 g}\right\}$ is a basis of $H_{1}(S)$ if and only if $\boldsymbol{T}$ has an inverse with integer entries, and this is true if and only if $\operatorname{det} \boldsymbol{T}=$ \pm 1 . The lemma follows.

Corollary 2.6. Let $S$ be a closed surface of genus g. Let $A$ be a subgroup of $H_{1}(S)$ such that $\mathrm{sc}(x, y)=0$ for any $x, y \in A$. Then the rank of $A$ is at most $g$.

Proof. Let $r$ be the rank of $A$. There exists a basis $\left\{a_{1}, \cdots, a_{r}\right\}$ of $A$, a basis $\left\{b_{1}, \cdots, b_{2 g}\right\}$ of $H_{1}(S)$, and positive integers $k_{1}, \cdots, k_{r}$ such that $a_{i}=k_{i} b_{i}(i=1, \cdots, r)$. Obviously sc $\left(b_{i}, b_{j}\right)=0$ if $i, j \leqq r$. Therefore $\boldsymbol{B}$, the sc-matrix of $\left(b_{1}, \cdots, b_{2 g}\right)$, contains a zero $r \times r$ block. If $r>g$, then $\operatorname{det} \boldsymbol{B}=0$; but this is impossible by 2.5 .

The next proposition is an algebraic version of Theorem 2.1. 
Proposition 2.7. Let $W$ be a 1-acyclic 3-manifold and $S$ a closed surface of genus $g$ in int $W$. Denote by $U$ and $V$ the closures of the components of $W-S$ and by $i: S \rightarrow U, j: S \rightarrow V$ the inclusions. Let $A=\operatorname{Ker} i_{*}, B=\operatorname{Ker} j_{*}$. Then

(1) $H_{1}(S)=A \oplus B$ and either of $A, B$ has rank $g$;

(2) $i_{*} \mid B: B \rightarrow H_{1}(U)$ and $j_{*} \mid A: A \rightarrow H_{1}(V)$ are isomorphisms;

(3) if $x, y \in H_{1}(S)$ are either both in $A$ or both in $B$, then sc $(x, y)=0$.

Proof. Consider the Mayer-Vietoris sequence of $(W ; U, V)$ :

$$
\cdots \longrightarrow H_{1}(S) \stackrel{\alpha}{\longrightarrow} H_{1}(U) \oplus H_{1}(V) \longrightarrow H_{1}(W) \longrightarrow \cdots
$$

Since $H_{1}(W)=0, \alpha$ is an epimorphism. We will show that it is also one-to-one. Recall that $\alpha$ is defined by $\alpha(x)=\left(i_{*}(x),-j_{*}(x)\right)$. Take an $x \in \operatorname{Ker} \alpha=A \cap B$. Represent $x$ by a closed oriented 1-manifold $J \subset S$. Then $J$ bounds compact, oriented, properly embedded 2-manifolds $G^{\prime} \subset U$ and $G^{\prime \prime} \subset V . \quad G=G^{\prime} \cup G^{\prime \prime}$ is a closed orientable 2-manifold in int $W$. Let $G_{1}, \cdots, G_{n}$ be the components of $G$ and let $G_{r}^{\prime}=G^{\prime} \cap G_{r}$, $G_{r}^{\prime \prime}=G^{\prime \prime} \cap G_{r}, J_{r}=J \cap G_{r}=\partial G_{r}^{\prime}=\partial G_{r}^{\prime \prime}(r=1, \cdots, n)$. To prove that $x=0$ it suffices to show that each $J_{r}$ bounds a compact oriented 2-submanifold of $S$.

$G_{r}$ separates $W$. Let $M$ be the union of $G_{r}$ and a component of int $W-G_{r}$. Orient $M$ so that $\partial M=G_{r}^{\prime} \cup\left(-G_{r}^{\prime \prime}\right)$. Let $M^{\prime}=M \cap U$, $F=M \cap S$. Then $\mathrm{Bd} M^{\prime}=F \cup G_{r}^{\prime}$. If we orient $F$ so that $\partial M^{\prime}=$ $(-F) \cup G_{r}^{\prime}$, then $\partial F=\partial G_{r}^{\prime}=J_{r}$. We have thus shown that $\operatorname{Ker} \alpha=0$ and therefore $\alpha$ is an isomorphism. This proves (2) and the first part of (1) of our proposition. Obviously 1.1 implies (3), and (3) together with 2.6 imply the second part of (1).

Now we start proving Theorem 2.1. In the first step we will choose the homology classes for the simple closed curves which we want to construct: $a_{i}$ will be the homology class of $J_{i}, b_{i}$ of $K_{i}$. We work with a surface in limbo.

LEMma 2.8. Let $S$ be a closed oriented surface of genus $g$. Suppose that the group $H_{1}(S)$ is represented as a direct sum $A \oplus B$ so that $\mathrm{sc}(x, y)=0$ for any two elements $x, y \in H_{1}(S)$ which lie either both in $A$ or both in $B$. Then there exist bases $\left\{a_{1}, \cdots, a_{g}\right\}$ of $A$ and $\left\{b_{1}, \cdots, b_{g}\right\}$ of $B$ such that $\operatorname{sc}\left(a_{i}, b_{j}\right)=\delta_{i j}$ for each $i$ and $j$.

ADDENDUM 2.9. Let $0 \leqq r \leqq g$ and $0 \leqq s \leqq g$. Suppose that $\left\{a_{1}, \cdots, a_{r}\right\}$ is a basis of a direct summand of $A$ and $\left\{b_{1}, \cdots, b_{s}\right\}$ is $a$ basis of a direct summand of $B$ such that $\operatorname{sc}\left(a_{i}, b_{j}\right)=\delta_{i j}(i=1, \cdots, r$; 
$j=1, \cdots, s)$. Then we can find $a_{r+1}, \cdots, a_{g}, b_{s+1}, \cdots, b_{g}$ such that $a_{1}, \cdots, a_{g}, b_{1}, \cdots, b_{g}$ satisfy the conclusion of 2.8 .

Proofs of 2.8 and 2.9. First note that 2.6 implies that $A$ and $B$ have rank $g$. Assume that $r \geqq s$. If $r<g$, choose any elements $a_{r+1}^{\prime}, \cdots, a_{g}^{\prime}$ such that $\left\{a_{1}, \cdots, a_{r}, a_{r+1}^{\prime}, \cdots, a_{g}^{\prime}\right\}$ is a basis of $A$. Then set

$$
a_{i}=a_{i}^{\prime}-\sum_{k=1}^{s} \operatorname{sc}\left(a_{i}^{\prime}, b_{k}\right) a_{k}, \quad i=r+1, \cdots, g
$$

(if $s=0$, let $a_{i}=a_{i}^{\prime}$ ). Obviously $a_{1}, \cdots, a_{g}$ again form a basis of $A$ and sc $\left(a_{i}, b_{j}\right)=\delta_{i j}$ for $1 \leqq i \leqq g, 1 \leqq j \leqq s$.

Let us first consider the case $s=0$. Choose an arbitrary basis $\left\{b_{1}^{\prime}, \cdots, b_{g}^{\prime}\right\}$ of $B$. Let $C$ be the sc-matrix of $\left(a_{1}, \cdots, a_{g}, b_{1}^{\prime}, \cdots, b_{g}^{\prime}\right)$. Then

$$
C=\left[\begin{array}{rr}
\boldsymbol{O}_{g} & \boldsymbol{D} \\
-\boldsymbol{D}^{\prime} & \boldsymbol{O}_{g}
\end{array}\right]
$$

where $D$ is the $g \times g$ matrix whose $(i, j)$-entry is sc $\left(a_{i}, b_{j}^{\prime}\right)$. By 2.5, $\operatorname{det} \boldsymbol{C}=(\operatorname{det} \boldsymbol{D})^{2}=1$, therefore, $\boldsymbol{D}^{\prime}$ has an inverse $\boldsymbol{U}=\left(u_{i j}\right)$ with integer entries. Put

$$
b_{i}=\sum_{j=1}^{g} u_{i j} b_{j}^{\prime} \quad(i=1, \cdots, g)
$$

Let

$$
\boldsymbol{T}=\left[\begin{array}{ll}
\boldsymbol{I}_{g} & \boldsymbol{O}_{g} \\
\boldsymbol{O}_{g} & \boldsymbol{U}
\end{array}\right]
$$

Then, by 2.4 , the sc-matrix of $\left(a_{1}, \cdots, a_{g}, b_{1}, \cdots, b_{g}\right)$ is $\boldsymbol{T C} \boldsymbol{T}^{\prime}=\boldsymbol{J}_{2 g}$. This is what we wished to have.

If $s>0$ we work in the same way except that we do not start with an arbitrary basis $\left\{b_{1}^{\prime}, \cdots, b_{g}^{\prime}\right\}$ of $B$. Choose $b_{s+1}^{\prime \prime}, \cdots, b_{g}^{\prime \prime} \in B$ such that $b_{1}, \cdots, b_{s}, b_{s+1}^{\prime \prime}, \cdots, b_{g}^{\prime \prime}$ form a basis of $B$. Then set $b_{i}^{\prime}=b_{i}$ for $i=1, \cdots, s$ and

$$
b_{i}^{\prime}=b_{i}^{\prime \prime}-\sum_{k=1}^{s} \operatorname{sc}\left(a_{k}, b_{i}^{\prime \prime}\right) b_{k} \quad \text { for } \quad i=s+1, \cdots, g .
$$

Then $\left\{b_{1}^{\prime}, \cdots, b_{g}^{\prime}\right\}$ is a basis of $B$ and $\operatorname{sc}\left(a_{i}, b_{j}^{\prime}\right)=\delta_{i j}$ unless $i, j>s$. This means that the matrices $\boldsymbol{D}$ and $\boldsymbol{U}$, defined as above, have the form

$$
D=\left[\begin{array}{cc}
I_{s} & O \\
O & E
\end{array}\right], \quad U=\left[\begin{array}{cc}
I_{s} & O \\
O & V
\end{array}\right]
$$

where $\boldsymbol{E}$ is a $(g-s) \times(g-s)$ matrix and $\boldsymbol{V}=\left(\boldsymbol{E}^{\prime}\right)^{-1}$. Therefore, the defining formula 2.10 yields the a priori given $b_{i}$ for $i=1, \cdots, s$. 
Lemma 2.8 and its Addendum are proved.

We have chosen the homology classes of our future simple closed curves $J_{i}$ and $K_{i}$. Now we will show that the chosen homology classes can really be represented by simple closed curves.

Proposition 2.11. Let $S$ be a closed surface and $x \in H_{1}(S)$. Then there exist an oriented simple closed curve $J \subset S$ and a positive integer $n$ such that $x$ is the homology class of the 1-cycle $n J$.

This proposition obviously follows from.

LEMma 2.12. Let $S$ be a closed surface and $K \subset S$ a closed oriented 1-manifold. Then there exists a sequence $K^{(1)}, K^{(2)}, \cdots, K^{(m)}$ of closed oriented 1-submanifolds of $S$ such that

(1) $K^{(1)}=K$;

(2) $K^{(i+1)}$ is obtained from $K^{(i)}$ either by omitting a component of $K^{(i)}$ which separates $S$ or by piping together two components of $K^{(i)}(i=1, \cdots, m-1)$;

(3) any two components of $K^{(m)}$ are homologous in $S$.

Proof. We use induction on the number of components of $K$. If $K$ is connected, then there is nothing to prove. Suppose that 2.12 is true if $K$ has less than $n$ components $(n \geqq 2)$. Choose a closed oriented 1-manifold $K \subset S$ which has $n$ components, say $K_{1}, \cdots, K_{n}$. Denote by $T$ the 2-manifold obtained by cutting $S$ along $K$, and let $p: T \rightarrow S$ be the corresponding identification map. Let $L_{r 1}, L_{r 2}$ be the two components of $p^{-1}\left(K_{r}\right)(r=1, \cdots, n)$; orient them so that $p$ maps each of them onto $K_{r}$ in an orientation preserving way.

Case 1. Suppose that $T$ has a component $T_{0}$ with connected boundary; let for instance $\mathrm{Bd} T_{0}=L_{r i}$. Then $K_{r}$ separates $S$. By induction hypothesis, 2.12 holds for $K^{\prime}=K-K_{r}$. It obviously follows that 2.12 holds for $K$.

Case 2. Suppose that $T$ has a component $T_{0}$ which has more than two boundary components. Then $T_{0}$ can be oriented so that two of its boundary components, say $L_{r i}$ and $L_{s j}$, are oriented coherently with $T_{0}$. By $1.2, L_{r i}$ and $L_{s j}$ can be piped together along any properly embedded arc $A \subset T_{0}$. We claim that $r \neq s$. Indeed, $S$ is obtained from $T$ by sewing each $L_{k 1}$ to $L_{k 2}$ by an orientation preserving homeomorphism and hence, if $L_{k 1}$ and $L_{k 2}$ lie in the same component of $T$ and if $T$ is given any orientation, one of $L_{k 1}, L_{k 2}$ is oriented coherently and the other incoherently with $T$. It follows that we can pipe $K_{r}$ to $K_{s}$ along the arc $p(A)$. Denote by $K_{r}^{\prime}$ the oriented simple closed curve obtained by this piping. By induction 
hypothesis, 2.12 holds for $K^{\prime}=\left(K-K_{r}-K_{s}\right) \cup K_{r}^{\prime}$, hence it holds for $K$.

Case 3. Finally we consider the case when each component of $T$ is bounded by exactly two simple closed curves. If some component of $T$ can be oriented coherently with both its boundary components, then we prove as in Case 2 that 2.12 holds for $K$. Suppose that no component of $T$ can be oriented coherently with its boundary. Obviously $T$ has $n$ components, say $T_{1}, \cdots, T_{n}$. Let $S_{i}=p\left(T_{i}\right)$ $(i=1, \cdots, n)$. Since $n>1$, no $S_{i}$ is a closed surface and therefore each $S_{i}$ is bounded by two components of $K$. We may assume that the numbering has been chosen so that $\mathrm{Bd} S_{i}=K_{i} \cup K_{i+1}(i=1, \cdots$, $n-1)$ and $\mathrm{Bd} S_{n}=K_{n} \cup K_{1}$. Since no $T_{i}$ can be oriented coherently with its boundary, the same holds for $S_{i}$. This means that each $S_{i}$ can be oriented so that $\partial S_{i}=\left(-K_{i}\right) \cup K_{i+1}(i=1, \cdots, n-1)$. It follows that $K_{1} \sim K_{2} \sim \cdots \sim K_{n}$ in $S$. This concludes the proof of 2.12 .

Theorem 2.1 follows from 2.7 and the following proposition.

Proposition 2.13. Let $S$ be a closed oriented surface of genus $g$. Suppose that $H_{1}(S)$ is represented as a direct sum $A \oplus B$ so that sc $(x, y)=0$ for any $x, y \in H_{1}(S)$ that lie either both in $A$ or both in $B$. Then there exist oriented simple closed curves $J_{i}, K_{i}$ in $S$ $(i=1, \cdots, g)$ such that

(1) for each $i, J_{i} \cap K_{i}$ is a single point and sc $\left(J_{i}, K_{i}\right)=1$; if $i \neq j$, then $J_{i} \cap J_{j}=J_{i} \cap K_{j}=K_{i} \cap K_{j}=\varnothing$;

(2) the homology classes of $J_{1}, \cdots, J_{g}$ form a basis of $A$ and the homology classes of $K_{1}, \cdots, K_{g}$ form a basis of $B$.

ADDENDUM 2.14. Suppose that we are given elements $a_{1}, \cdots, a_{r^{\prime}} \in$ $A, b_{1}, \cdots, b_{s^{\prime}} \in B\left(0 \leqq r^{\prime} \leqq g, 0 \leqq s^{\prime} \leqq g\right)$ and oriented simple closed curves $J_{1}, \cdots, J_{r}, K_{1}, \cdots, K_{s} \subset S \quad\left(0 \leqq r \leqq r^{\prime}, 0 \leqq s \leqq s^{\prime}\right)$ such that the following conditions are satisfied:

(i) if $i \leqq \min (r, s), J_{i} \cap K_{i}$ is a single point; if $i \neq j$, then $J_{i} \cap J_{j}=\varnothing, J_{i} \cap K_{j}=\varnothing, K_{i} \cap K_{j}=\varnothing$ (each of these equalities is satisfied for all pairs $i, j$ for which it makes sense);

(ii) $a_{i}$ is the homology class of $J_{i}$ and $b_{j}$ is the homology class of $K_{j}(i=1, \cdots, r ; j=1, \cdots, s)$;

(iii) $\left\{a_{1}, \cdots, a_{r^{\prime}}\right\}$ is a basis of a direct summand of $A$ and $\left\{b_{1}, \cdots, b_{s^{\prime}}\right\}$ is a basis of a direct summand of $B$;

(iv) $\operatorname{sc}\left(a_{i}, b_{j}\right)=\delta_{i j}\left(i=1, \cdots, r^{\prime} ; j=1, \cdots, s^{\prime}\right)$. Then there exist oriented simple closed curves $J_{r+1}, \cdots, J_{g}, K_{s+1}, \cdots, K_{g}$ such that $J_{i}$ represents $a_{i}\left(i=r+1, \cdots, r^{\prime}\right), K_{j}$ represents $b_{j}\left(j=s+1, \cdots, s^{\prime}\right)$, and $J_{1}, \cdots, J_{g}, K_{1}, \cdots, K_{g}$ satisfy the conclusions of 2.13 . 
REMARK. It is not difficult to show that if $J_{1}, \cdots, J_{r}$ are disjoint oriented simple closed curves in $S$ such that $S-\left(J_{1} \cup \cdots \cup J_{r}\right)$ is connected, then the homology classes of these curves freely generate a direct summand of $H_{1}(S)$. Therefore, if $r^{\prime}=r$ and $s^{\prime}=s$, we can replace the condition (iii) above by: $a_{i} \in A, b_{i} \in B$, and $S-\left(J_{1} \cup \cdots \cup J_{r}\right)$, $S-\left(K_{1} \cup \cdots \cup K_{s}\right)$ are connected.

In the proof of 2.13 and 2.14 we shall need the following three lemmas. The proofs of 2.15 and 2.17 are easy and we omit them.

Lemma 2.15. Let $S$ be a surface, $J \subset S$ an oriented simple closed curve, and $L \subset S$ an oriented closed 1-manifold. Orient $S$ so that $n=\operatorname{sc}(J, L) \geqq 0$. Then $L$ is homologous to an oriented closed 1-manifold $K \subset S$ such that $J \cap K$ contains exactly $n$ points.

The $K$ of 2.15 may have to have more components than $L$. On the other hand, the following lemma is valid.

Lemma 2.16. Let $S$ be an oriented surface, $J \subset \operatorname{int} S$ an oriented simple closed curve, and $L \subset$ int $S$ an oriented closed 1-manifold such that $\operatorname{sc}(J, L)=1$. Then $L$ is homologous to an oriented simple closed curve $K \subset S$ such that $J \cap K$ contains exactly 1 point.

Proof. By 2.15 we may assume that $J \cap L$ has only one point. We will prove the lemma by induction on the number of components of $L$. If this number is 1 , we can take $K=L$. Suppose that 2.16 is true if $L$ has at most $n$ components $(n \geqq 1)$. Take an $L$ with $n+1$ components, say $L_{0}, L_{1}, \cdots, L_{n}$; let $L_{0}$ be the component which intersects $J$.

Denote by $T$ the 2-manifold obtained by cutting $S$ along all components of $L$ and let $p: T \rightarrow S$ be the corresponding identification map. Let $L_{i}^{\prime}, L_{i}^{\prime \prime}$ be the two boundary components of $T$ composing $p^{-1}\left(L_{i}\right)(i=0,1, \cdots, n)$. Orient $L_{i}^{\prime}$ and $L_{i}^{\prime \prime}$ so that $p$ maps each of them onto $L_{i}$ in an orientation preserving way. Obviously $p^{-1}(J)$ is an arc connecting $L_{0}^{\prime}$ and $L_{0}^{\prime \prime}$. Hence $L_{0}^{\prime \prime}$ and $L_{0}^{\prime}$ lie in the same component, $T_{0}$ say, of $T$. Clearly, $\mathrm{Bd} T_{0}$ intersects $p^{-1}\left(L-L_{0}\right)$. Changing the notation, if necessary, we can assume that $L_{1}^{\prime} \subset \mathrm{Bd} T_{0}$. Orient $T_{0}$ coherently with $L_{1}^{\prime}$. Then one of $L_{0}^{\prime}, L_{0}^{\prime \prime}$ is oriented coherently with $T_{0}$ and the other incoherently. Assume that $L_{0}^{\prime}$ is oriented coherently with $T_{0}$. Let $A \subset T_{0}$ be a properly embedded arc which misses $p^{-1}(J)$ and joins $L_{0}^{\prime}$ to $L_{1}^{\prime}$. By 1.2 we can pipe $L_{0}^{\prime}$ to $L_{1}^{\prime}$ along $A$. It follows that in $S$ we can pipe $L_{0}$ to $L_{1}$ along the $\operatorname{arc} p(A)$, whose interior misses $J \cup L$. This piping changes $L$ to a closed oriented 1-manifold, homologous to $L$, which still intersects $J$ at a single point and has only $n$ components. Therefore, the induction hypothesis implies that 2.16 holds for $L$. 
LEMma 2.17. Let $S$ be a closed surface of genus $g>0$ and let $J, K \subset S$ be two simple closed curves crossing each other at a single point. Denote by $T$ the surface obtained by cutting $S$ along $J$ and $K$ and let $p: T \rightarrow S$ be the corresponding identification map. Let $S^{\prime}$ be the closed surface obtained by attaching a disk to $T$ along the boundary curve $p^{-1}(J \cup K)$ of $T$; let $k: T \rightarrow S^{\prime}$ be the inclusion. Then $k_{*}$ is an isomorphism and $p_{*} k_{*}^{-1}: H_{1}\left(S^{\prime}\right) \rightarrow H_{1}(S)$ maps $H_{1}\left(S^{\prime}\right)$ isomorphically onto the direct summand of $H_{1}(S)$ which consists of homology classes of 1-cycles that have zero intersection numbers with both $J$ and $K$. Moreover, if we orient $S$ and $S^{\prime}$ so that $p$ preserves orientation, then $p_{*} k_{*}^{-1}$ preserves intersection numbers.

Proofs of 2.13 and 2.14. By 2.8 and 2.9 we can assume that $r^{\prime}=s^{\prime}=g$. We also assume that $r \geqq s$.

The proof is by induction on the genus of $S$. If this genus is 0 , there is nothing to prove. Suppose that 2.13 and 2.14 are true if the genus of $S$ is less than $g(g>0)$ and consider a situation with the genus of $S$ equal to $g$.

If $r>0$, then we already have $J_{1}$. If $r=0$, choose any oriented simple closed curve representing $a_{1}$ (it follows from 2.11 that one such exists) and call it $J_{1}$. If $s>0$ (and hence $r>0$ by our assumption), then we already have $K_{1}$. Suppose that $s=0$. Represent $b_{1}$ by a closed oriented 1-manifold $L$. By 2.15 we can assume that $L \cap\left(J_{2} \cup \cdots \cup J_{r}\right)=\varnothing$. Applying 2.16 to $S-\left(J_{2} \cup \cdots \cup J_{r}\right), J_{1}$, and $L$ we can find an oriented simple closed curve $K_{1} \sim L$ such that $J_{1} \cap K_{1}$ is a single point and $K_{1} \cap\left(J_{2} \cup \cdots \cup J_{r}\right)=\varnothing$.

We can therefore assume that we already have a "good" pair $J_{1}, K_{1}$, either preassigned or constructed as described above. Define $T, p, S^{\prime}$, and $k$ as in the statement of 2.17 , with $J_{1}$ and $K_{1}$ taking the roles of $J$ and $K$, respectively. It follows from 2.17 that $S^{\prime}$, $g^{\prime}=g-1, \quad A^{\prime}=k_{*} p_{*}^{-1}(A), \quad B^{\prime}=k_{*} p_{*}^{-1}(B), \quad a_{i}^{\prime}=k_{*} p_{*}^{-1}\left(a_{i}\right) \quad$ and $\quad b_{i}^{\prime}=$ $k_{*} p_{*}^{-1}\left(b_{i}\right) \quad(i=2, \cdots, g), J_{i}^{\prime}=k p^{-1}\left(J_{i}\right)$ and $K_{j}^{\prime}=k p^{-1}\left(K_{j}\right) \quad(i=2, \cdots, r$; $j=2, \cdots, s)$ satisfy the hypotheses of 2.13 and 2.14. By induction hypothesis we can represent each $a_{i}^{\prime}$ by an oriented simple closed curve $J_{i}^{\prime} \subset k(T) \subset S^{\prime}$ and each $b_{j}^{\prime}$ by an oriented simple closed curve $K_{j}^{\prime} \subset k(T) \subset S^{\prime}(i=r+1, \cdots, g ; j=s+1, \cdots, g)$ such that $J_{2}^{\prime}, \cdots$, $J_{g}^{\prime}, K_{2}^{\prime}, \cdots, K_{g}^{\prime}$ satisfy (1) of 2.13 . Let $J_{i}=p k^{-1}\left(J_{i}^{\prime}\right), K_{j}=p k^{-1}\left(K_{j}^{\prime}\right)$ $(i=r+1, \cdots, g ; j=s+1, \cdots, g)$. Then $J_{1}, \cdots, J_{g}, \quad K_{1}, \cdots, K_{g}$ satisfy the conclusions of 2.13 and 2.14.

We conclude this section with a proof of the following theorem.

THeOREM 2.18. Let $U$ be a cube with $g$ handles. Denote $\operatorname{Bd} U$ by $S$ and let $i: S \rightarrow U$ be the inclusion. Let $\left\{a_{1}, \cdots, a_{g}\right\}$ be any basis of $\operatorname{Ker} i_{*}$. Then we can represent each $a_{r}$ by an oriented simple 
closed curve $J_{r} \subset S(r=1, \cdots, g)$ such that $J_{1}, \cdots, J_{g}$ bound disjoint disks in $U$.

This result is implicitly contained in pp. 296-299 of [2]. But perhaps it is worth while stating and proving it explicitly. Let us first consider the following weaker lemma.

LEMma 2.19. Let $U, S$, and $i$ be as in 2.18. Then every $x \in$ Ker $i_{*}$ can be represented by a 1-cycle $n J$ where $n$ is a positive integer and $J \subset S$ is an oriented simple closed curve which bounds a disk in $U$.

Proof. We can assume that $x \neq 0$. Let $\left\{K_{1}, \cdots, K_{g}\right\}$ be a collection of oriented simple closed curves in $S$ which bound disjoint disks in $U$ and whose union does not separate $S$. Then the homology classes of $K_{1}, \cdots, K_{g}$ form a basis of $\operatorname{Ker} i_{*}$. Therefore, there exist integers $n_{1}, \cdots, n_{g}$ such that the 1-cycle $n_{1} K_{1}+\cdots+n_{g} K_{g}$ represents $x$. This obviously implies that $x$ can be represented by an oriented closed 1-manifold $K$ such that the components of $K$ bound disjoint disks in $U$. Therefore, 2.19 easily follows from 2.12 and the following obvious lemma.

LEMma 2.20. Let $U$ be a 3-manifold and let $L_{1}, L_{2}$ be oriented simple closed curves in $\mathrm{Bd} U$ bounding disjoint properly embedded disks $E_{1}$ and $E_{2}$, respectively, in $U$. Suppose that $L_{1}$ can be piped to $L_{2}$ along an arc $A \subset \mathrm{Bd} U$ and let $L$ be the simple closed curve obtained by this piping. Let $N$ be a neighborhood of $A$ in $U$ containing the "pipe" $L-\left(L_{1} \cup L_{2}\right)$. Then $L$ bounds a properly embedded disk $E \subset U$ which is contained in $E_{1} \cup E_{2} \cup N$.

Proof of 2.18. Suppose that 2.18 is false and take the smallest $g$ for which 2.18 fails. By 2.19, $g>1$.

Embed $U$ into $E^{3}$. Let $V=E^{3}-$ int $U$ and let $j: S \rightarrow V$ be the inclusion. Choose an orientation for $S$. It follows from 2.7, 2.8, and 2.9 that there exists a basis $\left\{b_{1}, \cdots, b_{g}\right\}$ of $\operatorname{Ker} j_{*}$ such that $\operatorname{sc}\left(a_{r}, b_{s}\right)=$ $\delta_{r s}(r, s=1, \cdots, g)$. By 2.19 we can represent $a_{1}$ by an oriented simple closed curve $J_{1} \subset S$ which bounds a properly embedded disk $D_{1} \subset U$. Obviously $U-D_{1}$ is connected.

Represent $a_{r}$ by an oriented closed 1-manifold $K_{r} \subset S$ and $b_{r}$ by an oriented closed 1-manifold $L_{r} \subset S(r=2, \cdots, g)$; by 2.15 we may assume that $J_{1} \cap K_{r}=J_{1} \cap L_{r}=\varnothing$. Choose compact, oriented, properly embedded 2-manifolds $F_{r} \subset U, G_{r} \subset V$ such that $\partial F_{r}=K_{r}, \partial G_{r}=$ $L_{r}$. If $F_{r}$ intersects $D_{1}$, we can put $F_{r}$ in general position with $D_{1}$, remove the part of $F_{r}$ which lies in a regular neighborhood of $D_{1}$, 
and then patch the resultant holes in $F_{r}$ by disjoint disks "parallel" to $D_{1}$. In this manner we can replace $F_{r}$ by another compact, oriented, properly embedded 2-manifold in $U$ such that it is bounded by $K_{r}$ and misses $D_{1}$. Therefore, we will assume that the originally chosen $F_{2}, \cdots, F_{g}$ were already disjoint from $D_{1}$.

Choose a regular neighborhood $N$ of $D_{1}$ in $E^{3}$ and let $U^{\prime}=$ $U-\operatorname{int} N, V^{\prime}=V \cup N, S^{\prime}=\mathrm{Bd} U^{\prime}=\mathrm{Bd} V^{\prime}$. Let $i^{\prime}$ and $j^{\prime}$ be the inclusions of $S^{\prime}$ in $U^{\prime}$ and $V^{\prime}$, respectively. $U^{\prime}$ is again a cube with handles ([2], 6.2). Let $T=S \cap S^{\prime}$. Then $S^{\prime}-$ int $T$ consists of two disjoint disks, which we denote by $D_{1}^{\prime}$ and $D_{1}^{\prime \prime}$. Let $J_{1}^{\prime}=\operatorname{Bd} D_{1}^{\prime}$, $J_{1}^{\prime \prime}=\operatorname{Bd} D_{1}^{\prime \prime}$. Orient $J_{1}^{\prime}$ and $J_{1}^{\prime \prime}$ so that $J_{1}^{\prime} \sim J_{1} \sim J_{1}^{\prime \prime}$ in $S$. Let $a_{r}^{\prime}, b_{r}^{\prime} \in H_{1}\left(S^{\prime}\right)$ be the homology classes of $K_{r}, L_{r}$, respectively $(r=$ $2, \cdots, g)$. Since $K_{r}$ bounds $F_{r}$ in $U^{\prime}$ and $L_{r}$ bounds $G_{r}$ in $V^{\prime}$ we have $a_{r}^{\prime} \in \operatorname{Ker} i_{*}^{\prime}, b_{r}^{\prime} \in \operatorname{Ker} j_{*}^{\prime}$. If we give $S^{\prime}$ the orientation which on $T$ agrees with the chosen orientation of $S$, then sc $\left(a_{r}^{\prime}, b_{s}^{\prime}\right)=\operatorname{sc}\left(K_{r}, L_{s}\right)=$ $\delta_{r s}$. Thus it follows from 2.5 that the $a_{r}^{\prime}$ and $b_{r}^{\prime}$ form a basis of $H_{1}\left(S^{\prime}\right)$ and therefore, $\left\{a_{2}^{\prime}, \cdots, a_{g}^{\prime}\right\}$ is a basis of $\operatorname{Ker} i_{*}^{\prime}$.

By supposition, 2.18 is true for cubes with $g-1$ handles. Thus there exist oriented simple closed curves $J_{2}^{\prime}, \cdots, J_{g}^{\prime} \subset S^{\prime}$ and disjoint properly embedded disks $D_{2}^{\prime}, \cdots, D_{g}^{\prime}$ in $U^{\prime}$ such that for each $r$ the following are true:

(a) $J_{r}^{\prime}$ is in the homology class $a_{r}^{\prime}$ and hence $J_{r}^{\prime} \sim K_{r}$ in $S^{\prime}$;

( b ) $J_{r}^{\prime}=\mathrm{Bd} D_{r}^{\prime}$.

Without loss of generality we can assume

( c ) $J_{r}^{\prime} \subset$ int $T$.

Note that $T-\left(J_{2}^{\prime} \cup \cdots \cup J_{g}^{\prime}\right)$ is connected.

It is easy to see that (a) above implies that $K_{r} \sim J_{r}^{\prime}+n_{r}^{\prime} J_{1}^{\prime}+$ $n_{r}^{\prime \prime} J_{1}^{\prime \prime}$ in $T$ for some integers $n_{r}^{\prime}, n_{r}^{\prime \prime}$. Hence $K_{r} \sim J_{r}^{\prime}+n_{r} J_{1}$ in $S$, where $n_{r}=n_{r}^{\prime}+n_{r}^{\prime \prime}$. We will therefore try to replace each $J_{r}^{\prime}+n_{r} J_{1}$ by a homologous oriented simple closed curve bounding a disk in $U$.

Suppose that $n_{2} \neq 0$. Let for instance $n_{2}>0$. We can show, by the same argument as twice before, that it is possible to pipe $J_{2}^{\prime}$ to $J_{1}^{\prime}$ along an arc whose interior misses $J_{1}^{\prime} \cup J_{1}^{\prime \prime} \cup J_{2}^{\prime} \cup \cdots \cup J_{g}^{\prime}$. (If $n_{2}<0$, we pipe $J_{2}^{\prime}$ to $-J_{1}^{\prime}$ ) By this piping we obtain an oriented simple closed curve $J_{2}^{\prime \prime} ; 2.20$ implies that $J_{2}^{\prime \prime}$ bounds a properly embedded disk $D_{2}^{\prime \prime} \subset U$ which is disjoint from $D_{1}, D_{3}^{\prime}, \cdots, D_{g}^{\prime}$. We replace $J_{2}^{\prime}$ by $J_{2}^{\prime \prime}$ and $D_{2}^{\prime}$ by $D_{2}^{\prime \prime}$. Now we have $K_{2} \sim J_{2}^{\prime \prime}+m_{2} J_{1}$, where $\left|m_{2}\right|=\left|n_{2}\right|-1$. It should now be clear how to finish the proof of 2.18 by induction on the number $\left|n_{2}\right|+\cdots+\left|n_{g}\right|$.

3. Compact 3-submanifolds of acyclic 3-manifolds. In this section we will prove the following two theorems.

Theorem 3.1. A compact connected 3-manifold $M$ whose boundary 
has $m$ components $(m>0)$ is subacyclic if and only if it satisfies the following conditions (1), (2), and either $\left(3^{\prime}\right)$ or $\left(3^{\prime \prime}\right)$ :

(1) $M$ is orientable;

(2) $H_{1}(M)$ is free;

( $\left.3^{\prime}\right) H_{2}(M)$ is free of rank $m-1$;

$\left(3^{\prime \prime}\right) \quad H_{1}(\mathrm{Bd} M) \rightarrow H_{1}(M)$ is onto.

THEOREM 3.2. Let $M$ be a compact, connected, subacyclic 3-manifold and $J$ a closed oriented 1-manifold lying in a boundary component $S$ of $M$. Let $F$ be an oriented surface and $h: \partial F \rightarrow J$ an orientation preserving homeomorphism. Then the polyhedron $P=F \cup{ }_{h} M$ can be embedded in an acyclic 3-manifold if and only if $J$ satisfies one of the following two conditions.

(1) The homology class of $J$ in $M$ is a basic element of $H_{1}(M)$.

(2) There exist compact 2-submanifolds $G, H \subset S$ such that $G \cup H=S, G \cap H=J$, and there exists an orientation of $S$ such that $\partial G=-\partial H=J$.

The proof of 3.1 in one direction is quite easy. Suppose that $M$ lies in an open acyclic 3-manifold $W$. Then $M$ is orientable. Let $V$ be the closure of $W-M$. The Mayer-Vietoris sequence of $(W ; M, V)$ contains the following subsequence

$$
0 \longrightarrow H_{1}(\mathrm{Bd} M) \longrightarrow H_{1}(M) \oplus H_{1}(V) \longrightarrow 0 \text {. }
$$

It follows that $H_{1}(M)$ is free and that $H_{1}(\mathrm{Bd} M) \rightarrow H_{1}(M)$ is onto.

The other direction of 3.1 will be proved by induction on $m$. First we show that the conditions $\left(3^{\prime}\right)$ and $\left(3^{\prime \prime}\right)$ of 3.1 are equivalent.

LEMMA 3.3. Let $M$ be a compact connected 3-manifold with $m$ boundary components and suppose that (1) and (2) of 3.1 are satisfied. Then $\left(3^{\prime}\right)$ and $\left(3^{\prime \prime}\right)$ of 3.1 are equivalent and they imply that $H_{2}(M, \mathrm{Bd} M) \approx H_{1}(M)$ and that the following sequence is split exact:

$$
0 \longrightarrow H_{2}(M, \mathrm{Bd} M) \stackrel{\partial_{*}}{\longrightarrow} H_{1}(\mathrm{Bd} M) \stackrel{i_{*}}{\longrightarrow} H_{1}(M) \longrightarrow 0
$$

(here $\partial_{*}$ and $i_{*}$ are the homomorphisms from the homology sequence of the pair $(M, \mathrm{Bd} M))$.

Proof. Since $H_{1}(M)$ and $H_{2}(M)$ are free, duality and the Universal Coefficient Theorem yield the following two relations

$$
H_{1}(M, \operatorname{Bd} M) \approx H^{2}(M) \approx H_{2}(M), H_{2}(M, \operatorname{Bd} M) \approx H^{1}(M) \approx H_{1}(M) .
$$

Consider the exact sequence for the reduced homology of the pair $(M, \mathrm{Bd} M)$ : 


$$
\begin{aligned}
\cdots \longrightarrow H_{2}(\mathrm{Bd} M) & \longrightarrow H_{2}(M) \longrightarrow H_{2}(M, \mathrm{Bd} M) \longrightarrow \\
& \longrightarrow H_{1}(\mathrm{Bd} M) \longrightarrow H_{1}(M) \longrightarrow H_{1}(M, \mathrm{Bd} M) \longrightarrow \tilde{H}_{0}(\mathrm{Bd} M) \longrightarrow 0 .
\end{aligned}
$$

Suppose that $H_{1}(\mathrm{Bd} M) \rightarrow H_{1}(M)$ is onto. Then $\tilde{H}_{0}(\mathrm{Bd} M) \approx H_{1}(M$, $\mathrm{Bd} M) \approx H_{2}(M)$ and hence $H_{2}(M)$ is free of rank $m-1$. Now suppose that $H_{2}(M)$ is free of rank $m-1$. Then $H_{1}(M, \mathrm{Bd} M) \rightarrow \widetilde{H}_{0}(\mathrm{Bd} M)$ is an epimorphism of free groups of the same rank and thus it is actually an isomorphism. It follows that $H_{1}(\mathrm{Bd} M) \rightarrow H_{1}(M)$ is onto.

We conclude the proof of 3.3 by showing that $\left(3^{\prime}\right)$ of 3.1 implies that $\partial_{*}: H_{2}(M, \mathrm{Bd} M) \rightarrow H_{1}(\mathrm{Bd} M)$ is one-to-one. It suffices to show that $H_{2}(\mathrm{Bd} M) \rightarrow H_{2}(M)$ is onto, and this follows from the fact that the image of $H_{2}(\mathrm{Bd} M) \rightarrow H_{2}(M)$ is free of rank $m-1$ (this is true for any 3-manifold $M$ which has exactly $m$ compact orientable boundary components) and that $H_{2}(M, \mathrm{Bd} M)$ is torsion free.

Now we start proving the remaining direction of 3.1.

LEMMA 3.4. Let $M$ be a compact connected 3-manifold having precisely $m$ boundary components and satisfying (1), (2), and (3') of 3.1. Suppose that there exists an oriented simple closed curve $K \subset \mathrm{Bd} M$ such that the homology class of $K$ in $M$ is a basic element of $H_{1}(M)$. Then $M$ can be embedded in a compact connected 3manifold $M^{\prime}$ which has again $m$ boundary components, again satisfies (1), (2), and (3') of 3.1, and whose boundary has smaller genus than $\mathrm{Bd} M$.

Proof. Denote by $S$ the component of Bd $M$ which contains $K$ and let $A$ be a regular neighborhood of $K$ in $S$. Let $M^{\prime}$ be the 3-manifold obtained by attaching a 2-handle $H$ to $M$ along $A$. Since $K$ does not separate $S, M^{\prime}$ has exactly $m$ boundary components and $\mathrm{Bd} M^{\prime}$ has smaller genus than $\mathrm{Bd} M$. Obviously $M^{\prime}$ is compact, connected, and orientable. By considering the Mayer-Vietoris sequence of $\left(M^{\prime} ; M, H\right)$ for reduced homology we can prove that $H_{1}\left(M^{\prime}\right)$ is free and $H_{2}\left(M^{\prime}\right) \approx H_{2}(M)$.

\section{LEMma 3.5. Theorem 3.1 is valid for $m=1$.}

Proof. Suppose that this is false. Among all 3-manifolds $M$ which are counterexamples to 3.1 for $m=1$ choose one whose boundary has the smallest genus. Because of 2.3, $H_{1}(M)$ is nontrivial. Choose a basic element $x \in H_{1}(M)$. It follows from $3.1\left(3^{\prime \prime}\right)$ and 2.11 that $x$ can be represented by a simple closed curve $K \subset \mathrm{Bd} M$. But then 3.4 yields a 3 -manifold $M^{\prime}$ which is a counterexample to 3.1 for $m=1$ and whose boundary has smaller genus than $\mathrm{Bd} M$. This contradicts our choice of $M$. 
Lemma 3.6. Theorem 3.1 is valid for $m=2$.

Proof. Suppose that the lemma is false. Choose a 3-manifold $M$ which is a counterexample to 3.1 for $m=2$ and whose boundary has the smallest possible genus. Our plan is to find a simple closed curve $K \subset \mathrm{Bd} M$ representing a basic element of $H_{1}(M)$; as in 3.5 this will lead to a contradiction.

Let $S^{\prime}$ and $S^{\prime \prime}$ be the two components of $\mathrm{Bd} M$, let $g^{\prime}$ be the genus of $S^{\prime}$ and $g^{\prime \prime}$ the genus of $S^{\prime \prime}$, and let $i^{\prime}: S^{\prime} \rightarrow M, i^{\prime \prime}: S^{\prime \prime} \rightarrow M$, $i$ : Bd $M \rightarrow M$ be inclusions. By 3.3, $H_{1}(M)$ has rank $g^{\prime}+g^{\prime \prime}$ and therefore $g^{\prime}+g^{\prime \prime}>0$.

\section{Sublemma 1. $\operatorname{Ker} i_{*}^{\prime}=\operatorname{Ker} i_{*}^{\prime \prime}=0$.}

Proof. Suppose that e.g. $\operatorname{Ker} i_{*}^{\prime} \neq 0$. Since $H_{1}(M)$ is free, $\operatorname{Ker} i_{*}^{\prime}$ is a direct summand of $H_{1}\left(S^{\prime}\right)$. Therefore, it follows from 2.11 that there exists a nonseparating oriented simple closed curve $J \subset S^{\prime}$ such that $J \sim 0$ in $M$. Let $K \subset S^{\prime}$ be a simple closed curve intersecting $J$ transversely at exactly one point. Choose an orientation for $M$, orient $\mathrm{Bd} M$ coherently with $M$, and then orient $K$ so that $\operatorname{sc}(J, K)=1$.

We claim that $K$ represents a basic element of $H_{1}(M)$. Suppose that for some oriented closed 1-manifold $L \subset M$ and for some positive integer $n, K$ is homologous to $n L$ in $M$. Since $M$ satisfies $\left(3^{\prime \prime}\right)$ of 3.1 we can assume that $L \subset \mathrm{Bd} M$. Then $K-n L$ is a 1-cycle in Bd $M$, homologous to 0 in $M$. By 1.1, $1-n \operatorname{sc}(J, L)=\operatorname{sc}(J, K-n L)=$ 0 . Hence $n=1$ and consequently $K$ represents a basic element of $H_{1}(M)$. As we know, this leads to a contradiction and hence our supposition above must be wrong. Sublemma 1 is proved.

Identify $H_{1}(\mathrm{Bd} M)$ with $H_{1}\left(S^{\prime}\right) \oplus H_{1}\left(S^{\prime \prime}\right)$ and let $p^{\prime}: H_{1}(\mathrm{Bd} M) \rightarrow$ $H_{1}\left(S^{\prime}\right), p^{\prime \prime}: H_{1}(\mathrm{Bd} M) \rightarrow H_{1}\left(S^{\prime \prime}\right)$ be natural projections.

Sublemma 2. The compositions

$$
p^{\prime} \partial_{*}: H_{2}(M, \mathrm{Bd} M) \longrightarrow H_{1}\left(S^{\prime}\right), \quad p^{\prime \prime} \partial_{*}: H_{2}(M, \mathrm{Bd} M) \longrightarrow H_{1}\left(S^{\prime \prime}\right)
$$

are monomorphisms and hence $g^{\prime}=g^{\prime \prime}$.

Proof. Let $x \in H_{2}(M, \mathrm{Bd} M)$ be such that $p^{\prime} \hat{\partial}_{*}(x)=0$. Then $i_{*}^{\prime \prime} p^{\prime \prime} \partial_{*}(x)=i_{*}^{\prime} p^{\prime} \partial_{*}(x)+i_{*}^{\prime \prime} p^{\prime \prime} \partial_{*}(x)=i_{*} \partial_{*}(x)=0$. This equality and Sublemma 1 imply that $p^{\prime \prime} \partial_{*}(x)=0$. Therefore, $\partial_{*}(x)=0$ and hence, by 3.3, $x=0$. Similarly we show that $p^{\prime \prime} \partial_{*}$ is one-to-one.

By 3.3, $H_{2}(M, \mathrm{Bd} M)$ has rank $g^{\prime}+g^{\prime \prime}$. Since $p^{\prime} \partial_{*}$ is one-to-one, $g^{\prime}+g^{\prime \prime} \leqq 2 g^{\prime}$; similarly, $g^{\prime}+g^{\prime \prime} \leqq 2 g^{\prime \prime}$. Hence $g^{\prime}=g^{\prime \prime}$.

Denote $g^{\prime}=g^{\prime \prime}$ by $g$. 
Sublemma 3. There exist oriented simple closed curves $J, K \subset$ Bd $M$, one lying in $S^{\prime}$ and the other in $S^{\prime \prime}$ and neither homologous to 0 in $\mathrm{Bd} M$, and there exists a positive integer $r$ such that $J+r K \sim 0$ in $M$.

Proof. Choose a basis $\left\{a_{1}^{\prime}, \cdots, a_{2 g}^{\prime}\right\}$ of $H_{1}\left(S^{\prime}\right)$, a basis $\left\{b_{1}^{\prime}, \cdots, b_{2 g}^{\prime}\right\}$ of $E^{\prime}=p^{\prime} \partial_{*} H_{2}(M, \mathrm{Bd} M)$, and positive integers $n_{1}^{\prime}, \cdots, n_{2 g}^{\prime}$ such that $b_{j}^{\prime}=n_{j}^{\prime} a_{j}^{\prime}(j=1, \cdots, 2 g)$. Let $n^{\prime}$ be the greatest common divisor of $n_{1}^{\prime}, \cdots, n_{2 g}^{\prime}$.

Suppose that $n^{\prime}=1$. Then $E^{\prime}$ contains a basic element of $H_{1}\left(S^{\prime}\right)$. Indeed; suppose that no element of $E^{\prime}$ is basic for $H_{1}\left(S^{\prime}\right)$. Then there exists a prime $q$ such that $E^{\prime} \subset q H_{1}\left(S^{\prime}\right)$ (see e.g. [1], 5.1.1). Since $n^{\prime}=1$ there is an $s(1 \leqq s \leqq 2 g)$ such that $n_{s}^{\prime}$ is not divisible by $q$. Then, as $a_{s}^{\prime}$ is a basic element of $H_{1}\left(S^{\prime}\right), b_{s}^{\prime}=n_{s}^{\prime} a_{s}^{\prime}$ is not equal to $q x$ for any $x \in H_{1}\left(S^{\prime}\right)$ and this contradicts our previous conclusion. Thus there really exists a $b^{\prime} \in E^{\prime \prime}$ which is a basic element of $H_{1}\left(S^{\prime}\right)$. Let $b^{\prime \prime}=p^{\prime \prime} \partial_{*}\left(p^{\prime} \partial_{*}\right)^{-1}\left(b^{\prime}\right) \in H_{1}\left(S^{\prime \prime}\right)$. By Sublemma $2, b^{\prime \prime} \neq 0$. It follows from 2.11 that there exist oriented simple closed curves $J \subset S^{\prime}$, $K \subset S^{\prime \prime}$ and a positive integer $r$ such that $J$ represents $b^{\prime}$ and $r K$ represents $b^{\prime \prime}$. Since $\left(b^{\prime}, b^{\prime \prime}\right)=\partial_{*}\left(p^{\prime} \partial_{*}\right)^{-1}\left(b^{\prime}\right) \in \operatorname{Ker} i_{*}, J+r K \sim 0$ in $M$. Thus Sublemma 3 is true in this case.

Now suppose that $n^{\prime}>1$. For each $j$ let $b_{\jmath}^{\prime \prime}=p^{\prime \prime} \partial_{*}\left(p^{\prime} \partial_{*}\right)^{-1}\left(b_{j}^{\prime}\right) \epsilon$ $H_{1}\left(S^{\prime \prime}\right)$; let $a_{j}^{\prime \prime}$ be the basic element of $H_{1}\left(S^{\prime \prime}\right)$ and $n_{j}^{\prime \prime}$ the positive integer such that $b_{j}^{\prime \prime}=n_{j}^{\prime \prime} a_{j}^{\prime \prime}$. Let $n^{\prime \prime}$ be the greatest common divisor of $n_{1}^{\prime \prime}, \cdots, n_{2 g}^{\prime \prime}$. If $n^{\prime \prime}=1$ we show as above that Sublemma 3 is valid. Suppose that $n^{\prime \prime}>1$. We will show that this leads to a contradiction. Choose a prime divisor $q$ of $n^{\prime \prime}$. By 2.5 the determinant of the intersection number matrix of $\left(a_{1}^{\prime}, \cdots, a_{2 q}^{\prime}\right)$ is equal to 1. Therefore, there exists an entry of this matrix, say $\operatorname{sc}\left(a_{s}^{\prime}, a_{t}^{\prime}\right)$, which is not divisible by $q$. Note that each pair $\left(b_{j}^{\prime}, b_{j}^{\prime \prime}\right)$ lies in Ker $i_{*}$. Therefore, 1.1 implies that sc $\left(\left(b_{s}^{\prime}, b_{s}^{\prime \prime}\right),\left(b_{t}^{\prime}, b_{t}^{\prime \prime}\right)\right)=0$ and hence $\operatorname{sc}\left(b_{s}^{\prime}, b_{t}^{\prime}\right)=-\mathrm{sc}\left(b_{s}^{\prime \prime}, b_{t}^{\prime \prime}\right)$. Since the number $n_{s}^{\prime} n_{t}^{\prime} \mathrm{sc}\left(a_{s}^{\prime}, a_{t}^{\prime}\right)=$ $-n_{s}^{\prime \prime} n_{t}^{\prime \prime} \operatorname{sc}\left(a_{s}^{\prime \prime}, a_{t}^{\prime \prime}\right)$ is divisible by $q$ and sc $\left(a_{s}^{\prime}, a_{t}^{\prime}\right)$ is not, one of $n_{s}^{\prime}, n_{t}^{\prime}$, say $n_{s}^{\prime}$, is divisible by $q$. Let for instance $n_{s}^{\prime}=q k^{\prime}, n_{s}^{\prime \prime}=q k^{\prime \prime}$. Put $a^{\prime}=k^{\prime} a_{s}^{\prime}, a^{\prime \prime}=k^{\prime \prime} a_{s}^{\prime \prime}$. Then the basic element $\left(p^{\prime} \partial_{*}\right)^{-1}\left(b_{s}^{\prime}\right)$ of $H_{2}(M, \mathrm{Bd} M)$ is mapped by $\partial_{*}$ to $\left(b_{s}^{\prime}, b_{s}^{\prime \prime}\right)=q\left(a^{\prime}, a^{\prime \prime}\right)$. This contradicts the fact that $\partial_{*}$ embeds $H_{2}(M, \mathrm{Bd} M)$ as a direct summand into $H_{1}(\mathrm{Bd} M)$. Sublemma 3 is proved.

We conclude the proof of 3.6 with

Sublemma 4. $K$ represents a basic element of $H_{1}(M)$.

Proof. Assume that $J \subset S^{\prime}, K \subset S^{\prime \prime}$. Let $u \in H_{1}\left(S^{\prime}\right), v \in H_{1}\left(S^{\prime \prime}\right)$ be the homology classes of $J, K$, respectively; let $x=\partial_{*}^{-1}(u, r v) \in$ 
$H_{2}(M, \mathrm{Bd} M)$. Since $u$ is a basic element of $H_{1}\left(S^{\prime}\right), x$ is basic for $H_{2}(M, \mathrm{Bd} M)$. Let $H_{2}(M, \mathrm{Bd} M)=A \oplus B$ where $A$ is the subgroup generated by $x$. Let $A^{\prime}$ be the subgroup of $H_{1}\left(S^{\prime}\right)$ generated by $u, A^{\prime \prime}$ the subgroup of $H_{1}\left(S^{\prime \prime}\right)$ generated by $v, B^{\prime}$ the smallest direct summand of $H_{1}\left(S^{\prime}\right)$ containing $p^{\prime} \partial_{*}(B)$, and $B^{\prime \prime}$ the smallest direct summand of $H_{1}\left(S^{\prime \prime}\right)$ containing $p^{\prime \prime} \partial_{*}(B)$. Then $H_{1}\left(S^{\prime}\right)=A^{\prime} \oplus B^{\prime}$ and $H_{1}\left(S^{\prime \prime}\right)=A^{\prime \prime} \oplus B^{\prime \prime}$.

We have to show that $i_{*}^{\prime \prime}(v)=i_{*}(0, v)$ is a basic element of $H_{1}(M)$. It follows from Sublemma 1 that $i_{*}^{\prime \prime}(v) \neq 0$. Suppose that there exists an integer $n>1$ and an element $z \in H_{1}(M)$ such that $i_{*}^{\prime \prime}(v)=$ nz. It follows from 3.3 that there exist elements $a \in H_{1}\left(S^{\prime}\right), \quad b \in$ $H_{1}\left(S^{\prime \prime}\right), y \in H_{2}(M, \mathrm{Bd} M)$ such that $z=i_{*}(a, b)$ and $(n a, n b-v)=\partial_{*}(y)$. Let $a=\alpha u+a_{0}, b=\beta v+b_{0}, y=\lambda x+y_{0}$, where $\alpha, \beta, \lambda$ are integers and $a_{0} \in B^{\prime}, b_{0} \in B^{\prime \prime}, y_{0} \in B$. Then we have $\partial_{*}(y)=\lambda \partial_{*}(x)+\partial_{*}\left(y_{0}\right)$ or $(n a, n b-v)=\lambda(u, r v)+\partial_{*}\left(y_{0}\right)$. Applying on both sides of this equation the natural projection $H_{1}(\mathrm{Bd} M) \rightarrow A^{\prime}$ we obtain $n \alpha u=\lambda u$; projecting to $A^{\prime \prime}$ yields $(n \beta-1) v=\lambda r v$. The former equation implies that $\lambda$ is divisible by $n$, which contradicts the latter equation. Sublemma 4 and Lemma 3.6 are proved.

The following lemma is a special case of Theorem 3.2.

Lemma 3.7. Let $M^{\prime}$ be a compact, connected, subacyclic 3-manifold, $S$ a boundary component of $M^{\prime}$, and $A \subset S$ a separating annulus. Let $M$ be the 3-manifold obtained by attaching a 2-handle to $M^{\prime}$ along $A$. Then $M$ is subacyclic.

Proof. Embed $M^{\prime}$ in a homology 3-sphere $\Sigma$. Let $U$ be the closure of the component of $\Sigma-S$ which intersects $M^{\prime}$. Denote by $V$ the 3-manifold obtained by attaching a 2-handle $H$ to $U$ along $A$. Then there exists a natural embedding of $M$ into $V$ and therefore in order to prove our lemma it suffices to show that $V$ is subacyclic. Obviously $V$ is orientable. Since $H_{2}(U)=0$ (by the already proved part of 3.1), the following is a section of the Mayer-Vietoris sequence of $(V ; U, H)$ for reduced homology:

$$
0 \longrightarrow H_{2}(V) \longrightarrow H_{1}(A) \longrightarrow H_{1}(U) \longrightarrow H_{1}(V) \longrightarrow 0 \text {. }
$$

As $A$ separates $S$ the homomorphism $H_{1}(A) \rightarrow H_{1}(U)$ is trivial. Hence $H_{2}(V) \approx H_{1}(A)$ and $H_{1}(V) \approx H_{1}(U)$. Since $\mathrm{Bd} V$ has two components it follows from 3.6 that $V$ is subacyclic.

We conclude the proof of 3.1 by proving

Lemma 3.8. Suppose that 3.1 holds for $m<k(k>2)$. Then 3.1 is true for $m=k$. 
Proof. Let $M$ be a compact, connected 3-manifold whose boundary has $k$ components and which satisfies conditions (1), (2), and (3') of 3.1 (with $m=k$ ). Let $J$ be a properly embedded arc in $M$ whose endpoints lie in different components of $\mathrm{Bd} M$. Let $N$ be a regular neighborhood of $J$ in $M$ and let $M^{\prime}$ be the closure of $M-N$. Then $M^{\prime}$ is a compact, connected, orientable 3-manifold with $k-1$ boundary components. Let $S$ be the component of $\mathrm{Bd} M^{\prime}$ which intersects $N$. We can think of $N$ as a 2-handle attached to $M^{\prime}$ along the annulus $A=M^{\prime} \cap N$, which separates $S$. By considering the MayerVietoris sequence of $\left(M ; M^{\prime}, N\right)$ for reduced homology we can prove that $M^{\prime}$ satisfies (2) and ( $\left.3^{\prime}\right)$ of 3.1 (with $m=k-1$ ). By the hypothesis of the lemma this implies that $M^{\prime}$ is subacyclic. Hence, by $3.7, M$ is subacyclic.

Proof of 3.2. We consider all possible situations with respect to the homological properties of $J$ in $M$. We divide these situations in two larger groups. First we consider

Case 1. Suppose that $J \sim 0$ in $S$. In this case there exists a unique pair of compact 2-submanifolds of $S$, say $G, H$, such that $G \cup H=S, G \cap H=J$. Indeed; choose a point $x_{0} \in S-J$ and let $G$ be the closure of the set of all points in $S-J$ that can be reached from $x_{0}$ by some arc in $S$ which misses $J$ or crosses $J$ an even number of times; let $H=S$ - int $G$. If at least one of $G, H$ is such that it cannot be oriented coherently with $J$, then the polyhedron $P$ cannot be embedded in any acyclic 3-manifold. Suppose that e.g. $G$ cannot be oriented coherently with $J$. Then $F \cup G \subset P$ is a nonorientable closed 2-manifold and therefore, as observed in the beginning of $\S 2, F \cup G$ cannot be embedded in any acyclic 3-manifold.

Now suppose that both $G$ and $H$ can be oriented coherently with $J$. Then there exists an orientation for $S$ such that, for the induced orientations in $G$ and $H, J=\partial G=-\partial H$. Give $S$ this orientation. Let $G_{1}, \cdots, G_{n}$ be the components of $G$. For $i=1, \cdots, n$ do the following. Let $K_{i}=h^{-1}\left(\mathrm{Bd} G_{i}\right) \subset \mathrm{Bd} F$. Choose a disk with holes $G_{i}^{\prime} \subset G_{i}$ such that $\mathrm{Bd} G_{i}^{\prime}=J_{i} \cup \mathrm{Bd} G_{i}$ where $J_{i}$ is a simple closed curve in int $G_{i}$. Similarly choose a disk with holes $F_{i}^{\prime \prime} \subset F$ such that Bd $F_{i}^{\prime \prime}=$ $K_{i} \cup K_{i}^{\prime}$ where $K_{i}^{\prime}$ is a simple closed curve in int $F$; let $F_{1}^{\prime \prime}, \cdots, F_{n}^{\prime \prime}$ be pairwise disjoint. Orient $J_{i}$ coherently with $G_{i}^{\prime}$ and $K_{i}^{\prime}$ coherently with $F_{i}^{\prime}$.

Let $C=S \times I$, where $I=[0,1]$, be an outer collar of $M$ on $S$ and let $M^{\prime}=M \cup C, S^{\prime}=S \times 1 \subset \mathrm{Bd} M^{\prime}(S \times 0$ is identified with $S$ in the natural way). Since $F_{i}^{\prime}$ is homeomorphic to $G_{i}^{\prime}$ there exists a proper embedding $h_{i}^{\prime}$ of $F_{i}^{\prime \prime}$ into $G_{i}^{\prime} \times I \subset C$ such that $h_{i}^{\prime}\left|K_{i}=h\right| K_{i}$ and $h_{i}^{\prime}\left(K_{i}^{\prime}\right)=J_{i}^{\prime}=J_{i} \times 1 \subset S^{\prime}$. In particular, choose a function $f_{i}: F_{i}^{\prime} \rightarrow$ 
$I$ such that $f_{i}\left(K_{i}\right)=0, f_{i}\left(K_{i}^{\prime}\right)=1, f_{i}\left(\right.$ int $\left.F_{i}^{\prime}\right)=\operatorname{int} I$; extend $h \mid K_{i}$ to a homeomorphism $h_{i}: F_{i}^{\prime} \rightarrow G_{i}^{\prime}$ and then set $h_{i}^{\prime}(x)=\left(h_{i}(x), f_{i}(x)\right) \in G_{i}^{\prime} \times I$ $\left(x \in F_{i}^{\prime \prime}\right)$.

Let $H^{\prime}=\left(H \cup \cup G_{i}^{\prime}\right) \times 1 \subset S^{\prime}, G^{\prime}=S^{\prime}-\operatorname{int} H^{\prime}, J^{\prime}=\bigcup J_{i}^{\prime}$. Orient $S^{\prime}$ and $J^{\prime}$ so that the natural homeomorphisms $S^{\prime} \rightarrow S, J_{i}^{\prime} \rightarrow J_{i}$ preserve orientations. Then $J^{\prime}=-\partial G^{\prime}=\partial H^{\prime}$. Denote by $F^{\prime \prime}$ the closure of $F-\bigcup F_{i}^{\prime}$ and let $h^{\prime}: \partial F^{\prime} \rightarrow J^{\prime}$ be defined by $h^{\prime}\left|K_{i}^{\prime}=h_{i}^{\prime}\right| K_{i}^{\prime}(i=1, \cdots$, $n)$. Then $h^{\prime}$ is an orientation reversing homeomorphism. There is an obvious embedding of $P$ into $P^{\prime}=F^{\prime} \cup_{n^{\prime}}, M^{\prime}$. Thus, if we can prove that $P^{\prime}$ can be embedded in some acyclic 3-manifold $W$, then $P$ can be embedded in $W$. Note that each component of $G^{\prime}$ has connected boundary and that this implies that $H^{\prime}$ is connected. This means that we have reduced our problem to the case when one of $G, H$, say $G$, is connected. If we apply the procedure described above to this situation, we reduce the problem to the case when $J$ is a separating simple closed curve in $S$.

Let us therefore assume that $J \subset S$ is a separating simple closed curve. Let $A$ be a regular neighborhood of $J$ in $S$. Denote by $M^{\prime}$ the 3-manifold obtained by attaching a 2-handle to $M$ along $A$. Obviously $P$ can be embedded in $M^{\prime}$. By $3.7, M^{\prime}$ is subacyclic and therefore $P$ can be embedded in some acyclic 3-manifold.

Case 2. Suppose that $J$ is not homologous to 0 in $S$.

If $J \sim 0$ in $M$, then $P$ certainly cannot be embedded in any acyclic 3-manifold. Suppose that there exists an embedding $i: P \rightarrow W$ where $W$ is an open acyclic 3-manifold. Let $U$ be the closure of the component of $W-i(S)$ which contains $i$ (int $M$ ) and let $V$ be the closure of the other component of $W-i(S)$. Then $i(J)$ bounds in both $U$ and $V$. But this contradicts 2.7 (1).

Now suppose that the homology class of $J$ in $M$ is equal to $k x$ for some integer $k>1$ and some nonzero $x \in H_{1}(M)$. In this case the image of $x$ under $H_{1}(M) \rightarrow H_{1}(P)$ is a nonzero element of order $k$ in $H_{1}(P)$. Since 3.1 implies that a compact subpolyhedron of an acyclic 3-manifold has free first homology group, $P$ cannot be embedded in any acyclic 3-manifold.

Finally suppose that $J$ represents a basic element of $H_{1}(M)$. Consider the Mayer-Vietoris sequence of $(P ; M, F)$ for reduced homology:

$$
\begin{aligned}
0 \longrightarrow H_{2}(M) & \longrightarrow H_{2}(P) \longrightarrow H_{1}(J) \stackrel{\alpha}{\longrightarrow} H_{1}(M) \oplus H_{1}(F) \\
& \stackrel{\beta}{\longrightarrow} H_{1}(P) \longrightarrow \widetilde{H}_{0}(J) \longrightarrow 0 .
\end{aligned}
$$

It is not difficult to prove that $\alpha$ embeds $H_{1}(J)$ as a direct summand 
into $H_{1}(M) \oplus H_{1}(F)$. Therefore, the homomorphism $H_{2}(P) \rightarrow H_{1}(J)$ is trivial and, as $H_{1}(M)$ and $H_{1}(F)$ are free, the image of $\beta$ is free. It follows that $H_{2}(P) \approx H_{2}(M)$ and that $H_{1}(P) \approx \operatorname{Im} \beta \oplus \widetilde{H}_{0}(J)$ is free. Identify $F$ with $F \times 0 \subset F \times I$. Give $\mathrm{Bd}(F \times I)$ the orientation induced by the orientation of $F$ and choose an orientation for $S$. Extend $h$ to an orientation reversing embedding $g:(\mathrm{Bd} F) \times I \rightarrow S$ and construct $V=(F \times I) \cup{ }_{g} M$. Then $V$ is a compact, connected, orientable 3-manifold containing $P$, and $P$ is a deformation retract of $V$. It follows that $H_{2}(V) \approx H_{2}(M)$ and that $H_{1}(V)$ is free. Suppose that $\mathrm{Bd} M$ has $m$ components. Then, by $3.1, H_{2}(V) \approx H_{2}(M)$ is free of rank $m-1$. This implies that $\mathrm{Bd} V$ has at most $m$ components. On the other hand, $\mathrm{Bd} V$ has at least as many components as $\mathrm{Bd} M$. Thus $V$ has exactly $m$ boundary components. Now it follows from 3.1 that $V$ is subacyclic. This concludes the proof of 3.2 .

\section{REFERENCES}

1. P. J. Hilton and S. Wylie, Homology Theory: An Introduction to Algebraic Topology, Cambridge Univ. Press, Cambridge, 1960.

2. C. D. Papakyriakopoulos, A reduction of the Poincaré conjecture to group theoretic conjectures, Ann. of Math., (2) 77 (1963), 250-305.

Received July 17, 1972. Section 2 of this paper contains some of the results of the author's Ph. D. thesis written at the University of Wisconsin under the supervision of Professor J. W. Cannon. The author thanks the referee for his comments and suggestions.

University of Ljubluana, LuUbluana, Yugoslavia 



\section{PACIFIC JOURNAL OF MATHEMATICS}

\section{EDITORS}

RICHARD ARENS (Managing Editor) University of California

Los Angeles, California 90024

R. A. Beaumon'T

University of Washington Seattle, Washington 98105
J. Dugundj1*

Department of Mathematics University of Southern California Los Angeles, California 90007

D. Gilbarg and J. Milgram Stanford University Stanford, California 94305

\section{ASSOCIATE EDITORS}

E. F. BECKENBACH

B. H. NeUMANN

F. WOLF

K. YOSHIDA

\section{SUPPORTING INSTITUTIONS}

UNIVERSITY OF BRITISH COLUMBIA CALIFORNIA INSTITUTE OF TECHNOLOGY UNIVERSITY OF CALIFORNIA MONTANA STATE UNIVERSITY UNIVERSITY OF NEVADA NEW MEXICO STATE UNIVERSITY OREGON STATE UNIVERSITY UNIVERSITY OF OREGON OSAKA UNIVERSITY

\section{UNIVERSITY OF SOUTHERN CALIFORNIA STANFORD UNIVERSITY UNIVERSITY OF TOKYO UNIVERSITY OF UTAH WASHINGTON STATE UNIVERSITY UNIVERSITY OF WASHINGTON AMERICAN MATHEMATICAL SOCIETY NAVAL WEAPONS CENTER}

* C. R. DePrima California Institute of Technology, Pasadena, CA 91109, will replace J. Dugundji until August 1974. 


\section{Pacific Journal of Mathematics}

\section{Vol. 49, No. $1 \quad$ May, 1973}

A. Bigard, Free lattice-ordered modules ...........................

Richard Bolstein and Warren R. Wogen, Subnormal operators in strictly cyclic

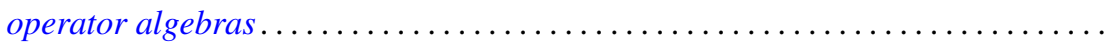

Herbert Busemann and Donald E. Glassco, II, Irreducible sums of simple

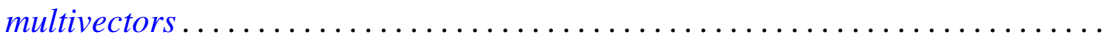

W. Wistar (William) Comfort and Victor Harold Saks, Countably compact groups

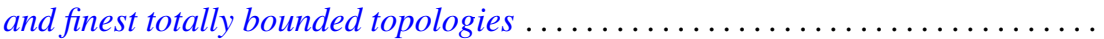

Mary Rodriguez Embry, Maximal invariant subspaces of strictly cyclic operator

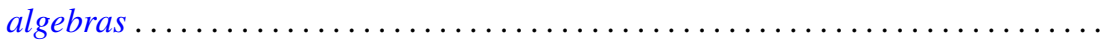

Ralph S. Freese and James Bryant Nation, Congruence lattices of semilattices......

Ervin Fried and George Grätzer, A nonassociative extension of the class of

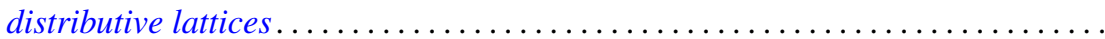

John R. Giles and Donald Otto Koehler, On numerical ranges of elements of locally

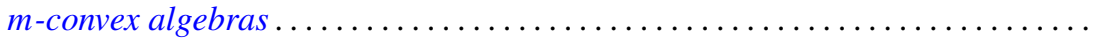

David A. Hill, On dominant and codominant dimension of $\mathrm{QF}-3$ rings ........ John Sollion Hsia and Robert Paul Johnson, Round and Pfister forms over $R(t) \ldots$ I. Martin (Irving) Isaacs, Equally partitioned groups . . . . . . . . . . . . . .

Athanassios G. Kartsatos and Edward Barry Saff, Hyperpolynomial approximation of solutions of nonlinear integro-differential equations.

Shin'ichi Kinoshita, On elementary ideals of $\theta$-curves in the 3-sphere and 2-links in the 4-sphere

Ronald Brian Kirk, Convergence of Baire measures

R. J. Knill, The Seifert and Van Kampen theorem via regular covering spaces ..

Amos A. Kovacs, Homomorphisms of matrix rings into matrix rings ..

Young K. Kwon, HD-minimal but no $H D$-minimal ..........

Makoto Maejima, On the renewal function when some of the mean renewal lifetimes are infinite

Juan José Martínez, Cohomological dimension of discrete modules over profinite groups.

W. K. Nicholson, Semiperfect rings with abelian group of units

Louis Jackson Ratliff, Jr., Three theorems on imbedded prime divisors of principal ideals.

Billy E. Rhoades and Albert Wilansky, Some commutants in $B(c)$ which are almost matrices

John Philip Riley Jr., Cross-sections of decompositions . . .

Keith Duncan Stroyan, A characterization of the Mackey uniformity $m\left(L^{\infty}, L^{1}\right)$ for

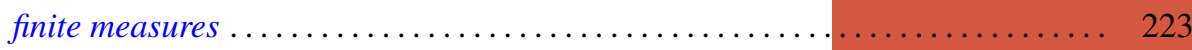

Edward G. Thurber, The Scholz-Brauer problem on addition chains . . . . . . . . . 229

Joze Vrabec, Submanifolds of acyclic 3-manifolds ............

Philip William Walker, Adjoint boundary value problems for compactified singular

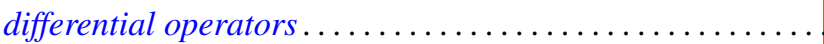

